\title{
ESTIMATES OF EIGENVALUES OF SCHRÖDINGER OPERATORS ON THE HALF-LINE WITH COMPLEX-VALUED POTENTIALS
}

\author{
ALEXANDRA ENBLOM
}

Abstract. Estimates for eigenvalues of Schrödinger operators on the half-line with complexvalued potentials are established. Schrödinger operators with potentials belonging to weak Lebesque's classes are also considered. The results cover those known previously due to R. L. Frank, A. Laptev and R. Seiringer [In spectral theory and analysis, vol. 214, Oper. Theory Adv. Appl., pag. 39-44; Birkhäuser/Springer Basel.]

Mathematics subject classification (2010): Primary 47E05, Secondary 35P15, 81Q12.

Keywords and phrases: Schrödinger operators, complex potentials, estimation of eigenvalues.

\section{REFERENCES}

[1] A. A. Abramov And A. Aslanyan, And E. B. Davies, Bounds on complex eigenvalues and resonances, J. Phys. A 34, 1 (2001), 57-72.

[2] J. Bergh AND J. LÖFSTRÖM, Interpolation spaces. An introduction, Grundlehren der Mathematischen Wissenschaften, no. 223, Springer-Verlag, Berlin, 1976.

[3] E. B. Davies AND J. NATH, Schrödinger operators with slowly decaying potentials, J. Comput. Appl. Math. 148, 1 (2002), 1-28.

[4] R. L. Frank And A. LAPteV And E. H. Lieb And R. Seiringer, Lieb-Thirring inequalities for Schrödinger operators with complex-valued potentials, Lett. Math. Phys. 77, 3 (2006), 309-316.

[5] R. L. FRANK AND A. LAPTEV AND R. SEIRINGER, A sharp bound on eigenvalues of Schrödinger operators on the half-line with complex-valued potentials. Spectral theory and analysis, Oper. Theory Adv. Appl., 214, 39-44, Birkhäuser/Springer Basel AG, Basel, 2011.

[6] J. B. KELLER, Lower bounds and isoperimetric inequalities for eigenvalues of the Schrödinger equation, J. Mathematical Phys. 2 (1961), 262-266.

[7] A. LAPTEV AND O. SAFRONOV, Eigenvalue estimates for Schrödinger operators with complex potentials, Comm. Math. Phys. 292, 1 (2009), 29-54.

[8] E. H. LIEB AND W. E. THIRRING, Inequalities for the Moments of the Eigenvalues of the Schrödinger Hamiltonian and Their Relation to Sobolev Inequalities, Studies in Mathematical Physics, 1976, Princeton Univ. Press, Princeton, NY, 269-303.

[9] R. O’NeIL, Convolution operators and L(p,q) spaces, Duke Math. J. 30 (1963), 129-142.

[10] M. Schechter, Spectra of partial differential operators, North-Holland Series in Applied Mathematics and Mechanics, 14, North-Holland Publishing Co., Amsterdam-London, American Elsevier Publishing Co., Inc., New York, 1971.

[11] H. TRIEBEL, Interpolation theory, function spaces, differential operators, VEB Deutscher Verlag der Wissenschaften, Berlin, 1978, 528. 